Trauma Berufskrankh 2007 - 9 [Suppl 2]:S158-S167 DOI 10.1007/s10039-006-1187-y

Online publiziert: 16. November 2006

(c) Springer Medizin Verlag 2006

J. M. Rueger · D. Briem · A. Rücker

Klinik für Unfall-, Hand- und Wiederherstellungschirurgie, Zentrum für

Operative Medizin, Universitätsklinikum Hamburg-Eppendorf, Hamburg

\title{
Azetabuläre Rekonstruktion
}

tion, dass höhere Arthroseraten durch die posttraumatische Entstehung einer avaskulären Nekrose des Femurkopfs bedingt sein sollen. Höhere Arthroseraten als die angegebenen $20 \%$ wurden als Folge einer insuffizienten Reposition betrachtet, d. h. dass der Femurkopf sich im weiteren Verlauf als „Stößel in einem Mörser“ selbst aufreibt $[9,19]$.

Der hohe Anspruch, Osteoarthroseraten nach einer Azetabulumfraktur von $\leq 20 \%$ durch die operative Behandlung zu erreichen, lässt sich nur erfüllen, wenn die in 0 Tab. 1 aufgelisteten und im Folgenden detaillierter erläuterten Voraussetzungen erfüllt sind.

\section{Adäquate Diagnostik}

[18] und Letournel u. Judet [15] für das Überleben des Gelenks nach Trauma und Rekonstruktion absolut ausschlaggebend (s. auch Wright et al. [25]).

Es ist zwar richtig, dass selbst bei anatomischer Rekonstruktion in den großen Serien von Letournel u. Judet [15], Matta $[16,17]$ und Matta et al. [18] bis zu 20\% der operativ behandelten Azetabulumfrakturen letztendlich in eine Arthrose übergingen. Die übrigen $80 \%$ zeigten jedoch bei anatomischer Reposition, d. h. also Gelenkstufen $<2 \mathrm{~mm}$, normale Überlebenszeiten 2 Jahre nach dem Trauma ohne die Entstehung einer Osteoarthrose. Dass 20\% aller betroffenen Gelenke auch bei anatomischer Reposition in einer Osteoarthrose enden, wurde von Letournel u. Judet [15], Matta $[16,17]$ und Matta et al. [18] auf das Ausmaß der primären Knorpelschädigung im Moment des Unfalls zurückgeführt (• Abb. 1). Klar abgelehnt wurde von ihnen die Argumenta-
Die Diagnostik der Azetabulumfraktur beruht auf der Trias:

- Abfragen/Eruieren des Unfallmechanismus und klinische Untersuchung

- Konventionelle Röntgenuntersuchung mittels 3 standardisierter Aufnahmen (a.-p., Ala-, Obturatoraufnahme, d. h. Judet-Projektionen, also $45^{\circ}$-Schrägaufnahmen [15])

- CT-Diagnostik - im Azetabulumbereich in $2-\mathrm{mm}$-Schritten - mit $2 \mathrm{D}$ Reformatierung mit/ohne $3 \mathrm{D}$-Rekonstruktion

\section{Anamnese/Unfallmechanismus}

Typischer Unfallmechanismus ist die axiale Stauchung über das Kniegelenk (z. B. Amaturenbrettverletzung), die in einer Krafteinleitung über das Femur und den Femurkopf zum Azetabulum resultiert. In Abhängigkeit von der Position des Femur- kopfs zum Azetabulum im Moment der Krafteinwirkung entstehen die klassischen Frakturformen entsprechend der Klassifikation nach Letournel u. Judet [15].

\section{Klinische Untersuchung}

Sie muss v. a. die Frage beantworten, ob eine offene Weichteilverletzung (eher selten bei der Azetabulumfraktur) oder ein Décollement im Sinne einer Morell-Lavalle-Läsion vorliegen ( $\mathbf{D}$ Abb. 2). Letztgenannte Verletzung kann in Abhängigkeit von ihrer Ausdehnung das weitere Vorgehen massiv beeinflussen, da der entstandene Hohlraum zwischen Haut und subkutanem Gewebe einerseits und den Muskelfaszien andererseits immer als bakteriell kontaminiert, also als offene Verletzung, zu betrachten ist. Entsprechend müssen im weiteren Vorgehen der Operationszeitpunkt und der Zugangsweg überdacht und festgelegt werden. Bei einem ausgedehntem Décollement ist möglicherweise sogar von einer operativen Versorgung abzusehen, um die Gefahr der Entwicklung einer Beckenosteitis $\mathrm{zu}$ vermeiden. Alle unsere Möglichkeiten, Weichteildefekte heutzutage mit modernen Methoden zu behandeln (z. B. VACTherapie), relativieren nicht die Aussage von Matta et al. [18]: „Besser eine posttraumatische Osteoarthrose nach der konservativen Behandlung einer Azetabulumfraktur als eine chronische Beckenosteitis nach der operativen Versorgung".

Von ebenso großer Bedeutung ist eine differenzierte neurologische Untersuchung im Rahmen der Primärdiagnostik. Insbesondere neurologische Defizite des $\mathrm{N}$. ischiadicus sind immer dann 
zu befürchten, wenn Frakturtypen auftreten, bei denen es zu einem Bruch des hinteren Walls \pm hinterem Pfeiler gekommen ist, und bei solchen Azetabulumfrakturen, die mit einer posterokranialen Hüftluxation/Hüftsubluxation vergesellschaftet sind. Nach Überprüfung des $\mathrm{N}$. ischiadicus ist es gerade bei Patienten mit Frakturen des hinteren Pfeilers/hinteren Walls notwendig, sie in der Operationsaufklärung darauf hinzuweisen, dass, auch wenn präoperativ klinisch-neurologisch kein Ischiadikusschaden festzustellen war und selbst bei völlig korrekter intraoperativer chirurgischer Technik, dennoch postoperativ mit einer höheren Ausfallrate des Nervs zur rechnen ist als bei Patienten, bei denen nur die anterioren Strukturen des Azetabulums betroffen sind. Ursache für diese größere Empfindlichkeit des N. ischiadicus nach einer Fraktur im Bereich des hinteren Pfeilers ist in einer primären Traumatisierung durch den Unfallmechanismus zu sehen, der noch keinen klinisch-neurologisch zu diagnostizierenden Nervenschaden hinterlassen hat. Intraoperativ, bei den wenigen Manipulationen des Nervs beim Kocher-Langenbeck-Zugang - dieser wird einmalig in seiner Lage in Bezug auf den M. quadratus femoris identifiziert und bleibt bei der gesamten weiteren Operation unberührt - sind in der Regel makroskopisch keine Einblutungen zu beobachten, dennoch scheint der Druck, der trotz aller Vorsicht über die Homann-Hebel in der Incisura ischiadica major intraoperativ hin und wieder auf den Nerv aufgebracht werden muss, auszureichen, einen durch den Unfallmechanismus vorgeschädigten Nerv komplett ausfallen zu lassen. Kommt es zu einer postoperativen Ischiadikus(teil)läsion, ist die Prognose in Bezug auf eine Regeneration im Lauf der Zeit eher ungünstig, wenn sich die Nervenfunktion nicht innerhalb der ersten 35 Tage postoperativ deutlich erholt.

\section{Konventionelles Röntgen}

Trotz aller Möglichkeiten der Computertomographie sollte insbesondere der weniger erfahrene Azetabulumchirurg nicht zuletzt aus autodidaktischen Gründen auf den klassischen 3 Röntgenbildern bestehen. Nur wenn es gelingt, die Frakturli-

Trauma Berufskrankh 2007 - 9 [Suppl 2]:S158-S167 DOI 10.1007/s10039-006-1187-y

(c) Springer Medizin Verlag 2006

\section{J. M. Rueger ·D. Briem·A. Rücker Azetabuläre Rekonstruktion}

\section{Zusammenfassung}

Der Erfolg der operativen Behandlung einer Azetabulumfraktur lässt sich an wenigen Kriterien messen: Größe der Stufen-/Spaltbildungen im Gelenk im unmittelbaren postoperativen $\mathrm{CT}$, der postoperativen knöchernen Heilung in anatomischer Position ohne sekundäre Dislokation, der Schmerzfreiheit und Beweglichkeit des Gelenks und dessen Langzeitüberleben im Verlauf, d. h. mindesten über das 2. Jahr nach der Operation hinaus, ohne dass es zu radiologischen oder klinischen Anzeichen der Entwicklung einer Koxarthrose kommt. Um diesen Behandlungserfolg zu erreichen, müssen die Fraktur präoperativ eindeutig verstanden und klassifiziert und standardisierte peri-, intra- und

\section{Acetabular reconstruction}

\section{Abstract}

Success in the treatment of an acetabular fracture can be measured on the basis of the following criteria: gap and step formation in the joint on a postoperative CT scan, secure bone healing postoperatively without secondary dislocation and with pain-free mobility, unrestricted weight bearing and longevity of the joint for more than two years after surgery without radiological and/or clinical signs of the onset of osteoarthritis. To attain this success the fracture has to be fully understood, correctly classified preoperatively and the treatment has to adhere to strict, standardized peri- and intraoperative protocols. postoperative Protokolle eingehalten werden, was nur dann möglich ist, wenn in der behandelnden Klinik die Grundvoraussetzungen personeller und logistischer Art für die operative Therapie von Azetabulumfrakturen gegeben sind. Diagnostik, Operationsvorbereitung, intraoperatives Vorgehen und Nachbehandlung inklusive der Prophylaxe von Komplikationen und deren Management bei diesen Verletzungen werden im vorliegenden Beitrag dargestellt.

\section{Schlüsselwörter}

Azetabulumfraktur - Diagnostik/Klassifikation · Zugangswege · Anatomische Reposition Nachbehandlung

Such treatment is only possible in hospitals which are sufficiently equipped in terms of experienced personnel and logistic organizations. The necessary steps that should be taken to achieve excellent results from the diagnosis to the preoperative set-up, surgical procedure, and postoperative treatment are presented in this paper.

\section{Keywords}

Acetabular fracture - Diagnosis/classification . Surgical approaches - Anatomical reduction . Postoperative treatment 


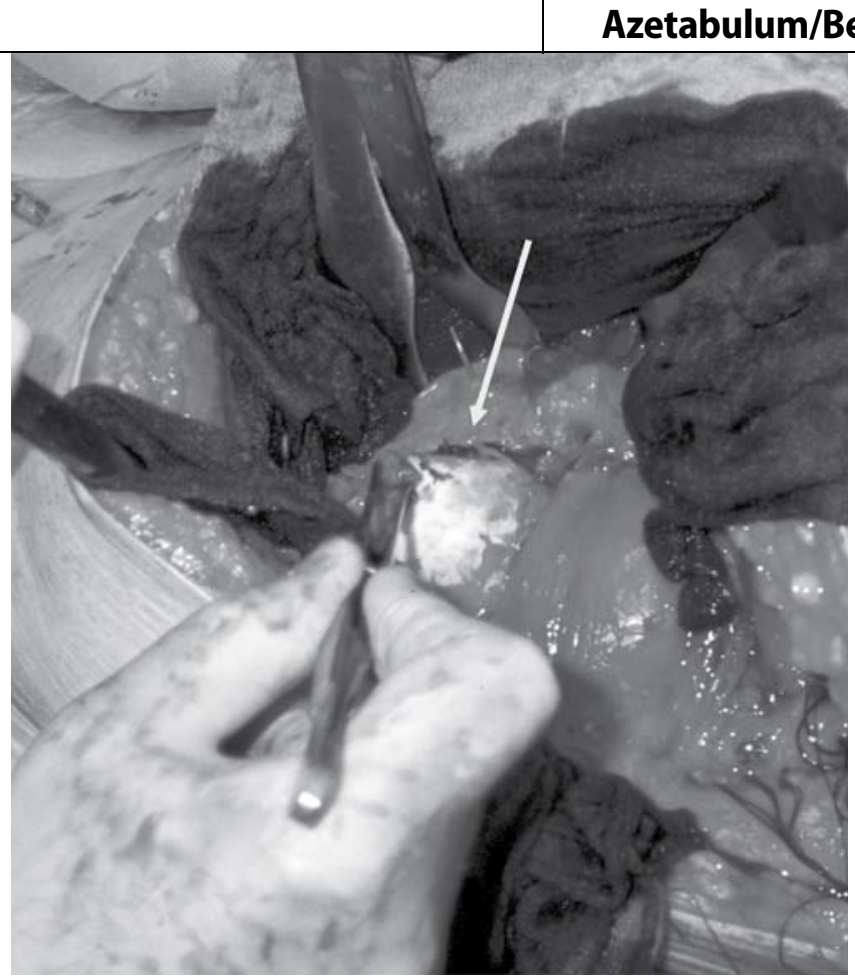

Abb. $1<47-$ jähriger Patient, hintere Wallfraktur, Kocher-Langenbeck-Zugang, intraoperative Fotografie in Bauchlage des Patienten, abgeplatteter Femurkopf (Pfeil) als Ausdruck des primären Knorpelschadens
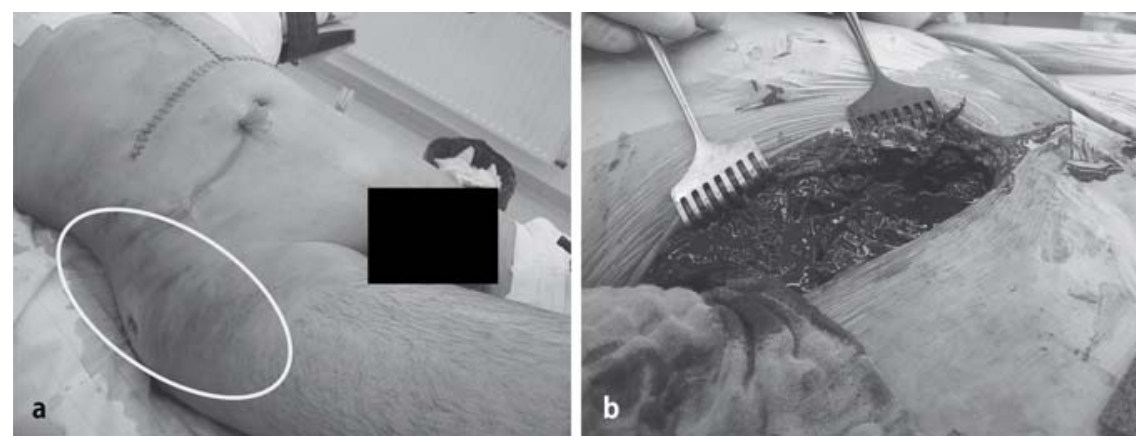

Abb. 2 × 29-jähriger Patient, a Zustand nach Hochrasanztrauma, Zustand nach Laparotomie, Tile-C3Fraktur mit Morell-Lavallé-Läsion rechtes Azetabulum, b Zustand nach posteriorem geradem Zugang zum rechten dorsalen Sakrum, massives Hämatom
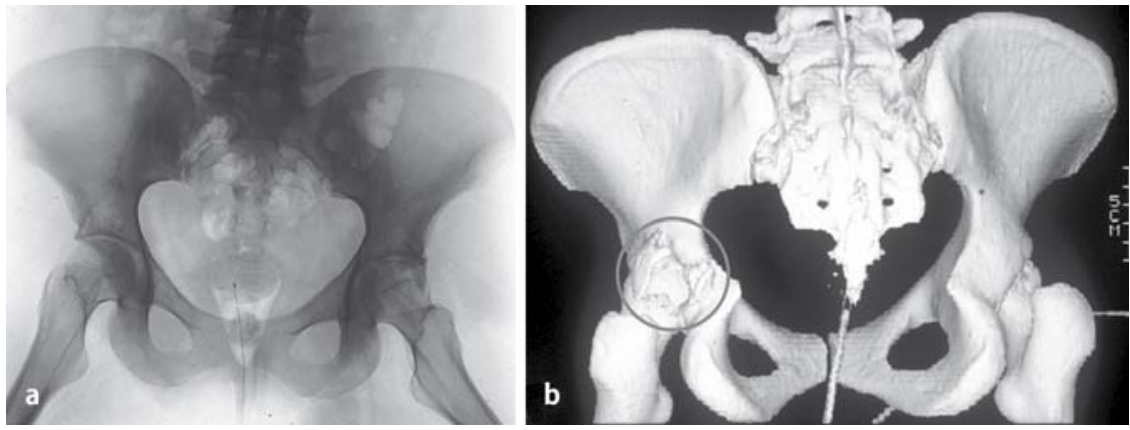

Abb. $3 \Delta$ 39-jähriger Patient, a Beckenübersichtsaufnahme, Fraktur nicht sicher erkennbar, b 3D-Rekonstruktion mit ausgedehnter Fraktur des hinteren Walls

nien der vorliegenden Azetabulumfraktur anhand dieser 3 Bilder sinnvoll auf ein Beckenplastikmodell zu übertragen, sollte man sich an die Klassifikation der Fraktur und die Entscheidungsfindungen:

a) Extension notwendig, b) Operationszeitpunkt und insbesondere Zugangsweg, Reihenfolge der Reposition und Fixationsschritte (s. unten)

heranwagen.
Erst wenn das Erkennen und die Klassifikation der Fraktur regelmäßig, zuverlässig und mit Erfolg - der sich nicht zuletzt in den postoperativen Ergebnissen widerspiegelt - gelingen, könnte man auf die Schrägaufnahmen verzichten und die Diagnose allein über die a.-p. Beckenaufnahme und die CT, möglicherweise sogar nur über die axialen Schnitte allein, erstellen.

\section{Computertomographie}

Sie muss das gesamte Becken darstellen, um möglichst zusätzliche Beckeninstabilitäten, insbesondere des hinteren Beckenrings, ausschließen zu können.

Die axialen Schnitte müssen des Weiteren insbesondere auf die folgenden Frakturen bzw. zusätzliche Informationen hin betrachtet werden:

- Vorderer Wall

- Vorderer Pfeiler

- Hinterer Wall

- Hinterer Pfeiler

- Querkomponente

- Ausmaß der Dislokation einzelner Komponenten

- Ausmaß der Inkongruenz zwischen Azetabulum und Femurkopf

- Marginale Impaktion

- Vorliegen freier Fragmente innerhalb des Gelenks

- Verletzung des Femurkopfs: Impression Absprengung/Fraktur (Pipkin)

- Frakturlinie(n) bis zum Sakroiliakalgelenk

- Knochenqualität

Die $2 \mathrm{D}$-Reformatierung erlaubt insbesondere in der koronaren Rekonstruktion den Vergleich mit dem nicht betroffenen Gelenk. Dagegen ermöglicht es insbesondere die sagittale Rekonstruktion, impaktierte Areale zu erkennen und das Ausmaß der Dislokation zwischen den Fragmenten zu beurteilen.

Die 3 D-Rekonstruktion sollte zuletzt betrachtet werden, da sie häufig nicht bei der Einteilung des Frakturtyps helfen kann. Sie ist dann sinnvoll zu verstehen, wenn bereits vorher, aufgrund der Betrachtung der konventionellen Röntgenaufnahmen und der $2 \mathrm{D}$-Schnitte, weitgehend Klarheit über den vorliegenden 
Frakturtyp besteht. Bei guter Rechnerqualität kann sie das Ausmaß der Verletzung als Gesamteindruck wiedergeben und so zu einem besseren „bildhaften“ Verständnis der Fraktur führen $[1,4,7,10,12,14$, 24]. In seltenen Fällen macht die $3 \mathrm{D}$-Rekonstruktion auch erst das Ausmaß der Verletzung wirklich deutlich (• Abb. 3).

\section{Eindeutige Klassifizierung}

Im klinischen Alltag stehen sich die Judet/Letournel- und die AO/OTA-Klassifikation der Azetabulumfrakturen gegenüber, wobei klar ersichtlich ist, dass Letztere auf Ersterer beruht. Im weiteren Verlauf soll daher nur auf das Klassifikationssystem von Letournel u. Judet [15] eingegangen werden, nicht zuletzt auch deshalb, weil es sich trotz seiner Komplexität hervorragend für die Einteilung der Frakturen eignet $[2,6]$ und das weitere Vorgehen operational festlegt.

Entsprechend des 2-Pfeiler-Konzepts repräsentieren Röntgenlinien in den Standardaufnahmen Strukturen des Azetabulums dar:

- Die ilioinguinale Linie repräsentiert den vorderen Pfeiler.

- Die ilioischiale Linie repräsentiert den hinteren Pfeiler.

- Die Linie des vorderen Walls repräsentiert die vordere azetabuläre $\mathrm{Ge}$ lenklippe.

- Die Linie des hinteren Walles repräsentiert die hintere azetabuläre $\mathrm{Ge}$ lenklippe.

- Die Tränenfigur repräsentiert die Fossa acetabuli.

- Der azetabuläre Dom repräsentiert das mechanische Hauptbelastungsareal des Azetabulums.

Eine Unterbrechung einer oder mehrerer dieser Linien bzw. die Kombination der Unterbrechung definierter Linien als Repräsentanten definierter Strukturen ist pathognomonisch für das Vorlegen bestimmter Frakturtypen. Dabei können diese im Sinn eines Ein-/Ausschlussverfahrens induktiv oder deduktiv abgeleitet werden.

Nach der Judet/Letournel-Klassifikation werden insgesamt 10 bestimmte Frakturtypen unterschieden: 5 einfache und 5 komplexe oder „combined fracture ty-

Tab. 1 Voraussetzungen für eine erfolgreiche operative Behandlung von Azetabulumfrakturen

1. Adäquate Diagnostik

2. Eindeutige Klassifizierung des Frakturtyps

3. Standardisierte Operationsbedingungen mit

Geeignetem Operationszeitpunkt

Präoperativer Abklärung auf das Vorliegen einer tiefen Bein-/ Beckenvenenthrombose

Perioperativer Antibiotikaprophylaxe

(Zephalosporin der 2. Generation)

Geeignetes Instrumentarium, d. h. Beckensieb(e)

Eingespielte Logistik (Anästhesie, Pflege, Operationsteam)

4. Anatomischer Zugangsweg entsprechend des Frakturtyps

5. Anatomische Rekonstruktion

6. Stabile Osteosynthese

7. Klares Nachbehandlungskonzept

\begin{tabular}{l} 
Postoperative Prophylaxe der Entwicklung heterotoper \\
Ossifikationen durch die Gabe von Indomethacin \\
\hline $\begin{array}{l}\text { Niedermolekulare Heparinisierung mindesten } \\
\text { bis zum Belastungsaufbau }\end{array}$ \\
\hline Postoperatives CT zur Qualitätskontrolle \\
\hline Frühmobilisation am 2. postoperativen Tag \\
\hline $\begin{array}{l}\text { Teilbelastung der operierten Extremität mit 5-10 kg } \\
\text { für 6 Wochen }\end{array}$ \\
\hline Verlaufskontrollen für insgesamt 2 Jahre \\
\hline In der Regel keine Materialentfernung \\
\hline
\end{tabular}

pes“. Wichtig ist, dass „einfacher Frakturtyp“ keineswegs „einfache Operation, einfache Operationstechnik und gute Prognose" bedeutet, teilweise ist sogar das Gegenteil der Fall.

\section{Indikationsstellung zur Operation}

Bei intakten Weichteilen ergibt sie sich aus der Beantwortung der folgenden, auf die Fraktur und den Patienten bezogenen Fragen:

1. Ist die Fraktur disloziert, findet sich eine Stufenbildung bzw. ist die Gelenkkongruenz aufgehoben?

2. Besteht eine Gelenkinkongruenz aufgrund einer marginalen Impaktion?

3. Finden sich freie, intraartikuläre Fragmente?

4. Wie groß sind die "roof arcs“ in den 3 Standardprojektionen, d. h. unterschreitet entweder auf der a.-p., der Ala- bzw. der Obturatoraufnahme der Winkel zwischen der Senkrechten auf das Zentrum des Femurkopfs und der nächstgelegenen Frakturlinie den Wert von $45^{\circ}$ (• Abb. 4) bzw. wie groß ist der mit dem CT gemessene subchondrale Bogen [22]?
5. Besteht eine sekundäre Kongruenz (symmetrische, dreidimensionale Durchmessererweiterung des Azetabulums. Selten: Nur beim Vorliegen einer 2-Pfeiler-Fraktur möglich)?

6. Wie alt ist die Fraktur, d. h. ist der Zeitraum zwischen Frakturereignis und geplantem Operationsdatum kleiner oder größer als 21 Tage? (Nachweislich sind die zu erzielenden Ergebnisse in Bezug auf Funktion und das radiologische Ergebnis in der Kohorte, die mehr als $21 \mathrm{Ta}$ ge nach dem Frakturereignis operiert wurde, schlechter als in der Patientengruppe, die bis zum 21. Tag nach dem Ereignis versorgt wurde [17].)

7. Wie alt ist der Patient bzw. wie gut ist seine Knochenqualität?

8. Welche Begleiterkrankungen bestehen, und ist der Patient narkosefähig?

9. Besteht für den Patienten in einem primären endoprothetischen Ersatz des Hüftgelenks eine echte Alternative [3]?

Ist eine der ersten 4 Fragen mit ,ja“ zu beantworten, ergibt sich daraus die Operationsindikation, die zunehmend auch 


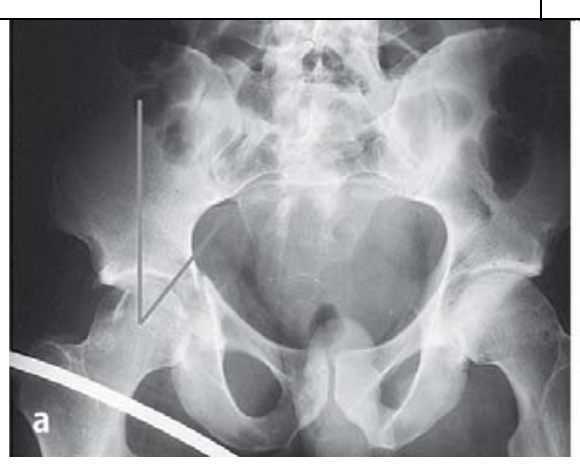

Abb. 4 வ 37-jähriger Patient, a transtektale Querfraktur mit hinterem Wall, a.-p. Aufnahme, ,roof arc measurement“: Senkrechte auf das Zentrum des Femurkopfs, verbunden mit einer die nächstgelegene, erkennbare Frakturlinie durchlaufenden Linie; in allen 3 Projektionen durchzuführen: Winkel zwischen Senkrechter und Referenzlinie in einer der 3 Projektionen $<45^{\circ}$ : Operationsindikation, b Ala-, c Obturatoraufnahme, Beteiligung des hinteren Walls sehr gut erkennbar (Pfeil)

bei Patienten bis zum und sogar über das 8 o. Lebensjahr hinaus gestellt werden kann, wobei selbstverständlich das biologische Alter des Patienten und eben die Alternative des primären endoprothetischen Gelenkersatzes kritisch abgewogen werden müssen. [Es gibt keinerlei evidenzbasierte Aussagen: „Rekonstruktion vs. Fraktur-TEP“. In Abhängigkeit von der Knochenqualität, dem biologischen Alter des Patienten und der Erfahrung des Operateurs können auch Azetabulumfrakturen hochbetagter Patienten ( $>85$ Jahre) sinnvoll osteosynthetisch mit ausgezeichneten Ergebnissen versorgt werden.]

\section{Standardisierte Operationsbedingungen}

Isolierte Azetabulumfrakturen stellen in der Regel - bis auf sehr wenige Ausnahmen - keine Notfallindikation dar. Selbst Luxationsfrakturen, die nach Reposition aufgrund der intrinsischen Instabilität reluxieren, sich aber mittels einer suprakondylären Femurdrahtextension halten lassen, sind kein „Notfall“. Prinzipiell ist die Indikation für eine Drahtextension, sei es suprakondylär am distalen Femur oder über den Tibiakopf, heute nur noch in den Fällen gegeben, bei denen entweder nach der Reposition der Femurkopf sofort reluxiert und dann die Femurkopfgelenkfläche auf einer Frakturebene des Azetabulums „reitet“ oder bei den azetabulären Frakturformen, die mit einer Querkomponente einhergehen, also:

- Querfrakturen,

- T-Frakturen und - vorstellbar -

- vordere Pfeiler- und hintere hemiquere Fraktur.
Bei Letzteren kann der Femurkopf ebenfalls, wie bei einer nichtretinierbaren Luxation, auf der Querkomponente „reiten“, also impaktieren. Die Begründung für die Extensionsbehandlung in diesen Situationen liegt darin, dass durch die Distraktion, das „Auflösen des Reitens“, eine weitere Knorpelschädigung vermieden werden kann.

(Isolierte) Azetabulumfrakturen sollten 3-5, maximal 7 Tage nach dem Ereignis operativ versorgt werden. Grundvoraussetzung sind eine eindeutige Klassifizierung, Verständnis der Frakturpersönlichkeit und Klarheit über den Zugangsweg, weiterhin ein eingespieltes Team mit der entsprechenden Logistik im Krankenhaus und einem standardisierten präund postoperativem Ablauf. Dazu gehören präoperativ

- Abführen,

- Venendoppler (Frage TVT) [8] und

- Blasenkatheter

sowie am Operationstag

- adäquate Lagerung mit der Möglichkeit der intraoperativen Durchleuchtung in den 3 Standardprojektionen, evtl. Inlet- und Outlet-Aufnahmen bei Beteiligung des Beckenrings.

Perioperativ sollte eine Antibiotikaprophylaxe mit einem Zephalosporin der 2. Generation vorgenommen werden. Die erste Gabe muss vor dem Hautschnitt erfolgen, mit einer Wiederholung der Dosis nach etwa 4-6 h Operationsdauer und Fortführung der „Prophylaxe“ für insgesamt 3 Tage (inklusive). Ein „cell saver" sollte angewandt werden, wobei realis- tisch gesehen werden muss, dass die Konserven, die sich am Operationstag einsparen lassen, in der Regel am 2.-3. postoperativen Tag auf der Station gegeben werden müssen - aufgrund des Abbaus der - am ehesten mechanisch - geschädigten, roten Blutkörperchen.

Selbstverständlich muss für die Operation ein spezielles Beckensieb (AOSynthes, Stryker, Matta-Set, - Abb.5) zum Einsatz kommen. Diese Siebe sind eine Grundvoraussetzung für die Operation aufgrund:

- der größeren mechanischen Stabilität der Becken- im Vergleich zu norma-

len Rekonstruktionsplatten,

- der auf den Sieben vorhandenen, langen Instrumente (3-Lippen-Bohrer, der für das empfohlene oszillierenden Bohren notwendig ist, Messgeräte, langer Kugelspieß mit Platte, usw.)

- der speziellen Repositionszangen wie Matta-/Faraboeuf-/Jungbluth-Zangen, koaxiale Zange der AO.

Wünschenswert sind weiterhin ein erfahrenes, mit den Beckeninstrumentarien vertrautes Operationspersonal, Kompetenz von Seiten des Durchleuchtenden und der anästhesiologischen Kollegen (intraoperative Volumengabe, Relaxation des Patienten bei der Hämatom-/Kallusausräumung und insbesondere bei der Reposition der Fragmente, der Pfeiler usw.).

\section{Operation}

\section{Anatomischer Zugangsweg}

Nach eindeutiger Klassifikation und Verstehen des Frakturtyps ist der nächstwich- 
tige Schritt die Entscheidung für den zu wählenden anatomischen Zugangsweg, der eine ideale Exposition der Fraktur und gleichzeitig deren sichere Reposition und Fixation erlaubt. Prinzipiell lassen sich alle Azetabulumfrakturen entweder über:

- den Kocher-Langenbeck-,

- den Ilioinguinalen oder

- den Erweiterten Iliofemoralen Zugang

operativ versorgen.

Mit zunehmender Erfahrung des Operateur ist zu beobachten, dass Frakturen, die zu Beginn der persönlichen Lernkurve über den Erweiterten Iliofemoralen Zugang behandelt wurden, sich auch über den ilioinguinalen Zugang, vereinzelt mit additiven, perkutanen Verschraubungen von dorsalen Fragmenten, adäquat behandeln lassen [11, 16]. Diese Art des Vorgehens führt zu einer deutlichen Reduktion des massiven Weichteiltraumas, das mit erweiterten Zugängen vergesellschaftet ist.

Unabhängig von der auf der persönlichen Erfahrung des Operateurs beruhenden Erweiterung der Indikationsstellung für den Ilioinguinalen Zugang im Vergleich zur Anwendung des Erweiterten Iliofemoralen Zugang wird von den erfahrenen Beckenchirurgen intensiv diskutiert, ob nicht prinzipiell, bei gegebener Indikation, anstelle eines einzeitigen erweiterten Zugangs nicht besser simultan oder zweizeitig der Ilioinguinale Zugang mit dem Kocher-Langenbeck-Zugang kombiniert verwandt werden sollte. Es herrscht Einigkeit darüber, dass erweiterte Zugänge wegen des assoziierten Weichteiltraumas auf Ausnahmen (z. B. transtektale, massiv dislozierte Querfrakturen, transtektale, massiv dislozierte T-Frakturen, jeweils ohne/mit Beteiligung des hinteren Walls) bzw. veraltete Frakturen ( $>14$ Tage) beschränkt werden sollten.

\section{Anatomische Reposition}

Nach Exposition der Fraktur müssen Frakturhämatom, eingeschlagenes Periost (auf dem R. superior bzw. im Bereich der quadrilateralen Oberfläche beim Ilioinguinalen Zugang) und neu gebildeter Kallus aus dem Gelenk entfernt werden. Ebenso

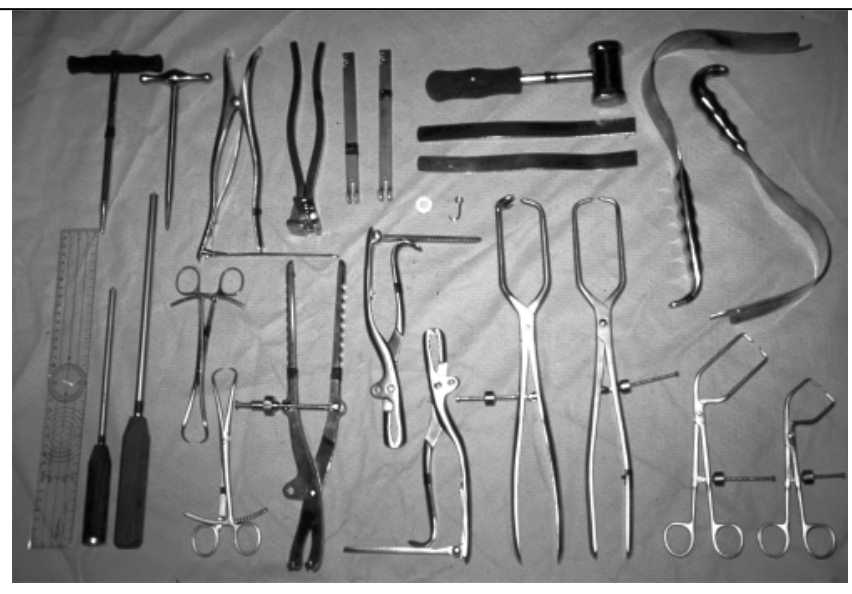

müssen freie Fragmente geborgen werden (nur beim Kocher-Langenbeck- bzw. Erweiterten Iliofemoralen Zugang möglich). Diese dürfen niemals verworfen, sondern müssen im Sinn eines dreidimensionalen Puzzles beim Wiederaufbau des Gelenks anatomisch korrekt eingepasst werden. Zur Fixation können bei nicht gelenkoberflächenbildenden Fragmenten des hinteren Walls entweder verlorene KirschnerDrähte (die umgebogen und in der Spongiosa des subchondralen Raums versenkt werden müssen) oder besser Titanschrauben (z. B. aus dem 2,oer Compact-System der AO) verwendet werden.

Sind die Fragmente mit Knorpel überzogen, müssen sie entweder mit 3,5-mmZugschrauben, wobei die Schraubenköpfe außerhalb des knorpeligen Überzugs des Gelenkes liegen müssen, fixiert oder anatomisch unter Beckenrekonstruktionsplatten eingepasst und indirekt über Kompression anatomisch gehalten werden. Selbstverständlich sollten im subchondralen Raum bzw. im Azetabulum selbst keine knöchernen Defekte belas

sen werden, sondern diese sind aufzufüllen (s. unten).

Bei der anatomischen Reposition marginal impaktierter Fragmente muss, bei der Wiederherstellung der Gelenkkongruenz, der Femurkopf zunächst in das ausgespülte, gesäuberte Gelenk reponiert sein. Er dient dann als Schablone, gegen den das marginal impaktierte Fragment vorsichtig, mittels eines kleinen Raspers mobilisiert und in Position gebracht wird. Nach der Fixation dieser Fragmente, in Abhängigkeit von ihrer Größe entweder mit verlorenen Kirschner-Drähten oder mit 2,0-mm-Titanzugschrauben, kann autologe Spongiosa für die Unterfütterung und Defektauffüllung in ausreichender Menge vom dorsalen Trochanter major gewonnen werden, um den Defekt innerhalb der Spongiosa des Azetabulums sicher aufzustopfen. Dieses Vorgehen ist problemlos sowohl beim Kocher-Langenbeck- als auch beim Erweiterten Iliofemoralen Zugang möglich (• Abb. 6).

\section{Stabile Osteosynthese}

Die sichere Stabilisierung mit 3,5-mm-Beckenrekonstruktionsplatten bzw. mit Zugschrauben ist die absolute Grundvoraussetzung für

- das Halten der anatomischen Reposition,

- die frühfunktionelle Therapie mit Krankengymnastik ab dem 2. postoperativen Tag und Teilbelastung, mit $5 \mathrm{~kg}$ beginnend, zum selben Zeitpunkt für insgesamt 6 Wochen,

- das Vermeiden einer sekundären Dislokation und

- die sichere knöcherne Heilung.

Intraoperativ, nach der anatomischen Reposition der Fragmente und - wenn nötig, aber eher selten - dem temporären Halten des Repositionsergebnisses mit Kirchner-Drähten (Stärken der Drähte zwischen 1,2 und 1,6 mm, wobei darauf zu achten ist, dass die temporär eingebrachten Kirschner-Drähte nicht im zukünftigen Plattenlager zu liegen kommen), müssen die Platten unbedingt anatomisch angeformt werden.

Es macht wenig Sinn bzw. setzt große Erfahrung voraus, über vorgebogene, an- 


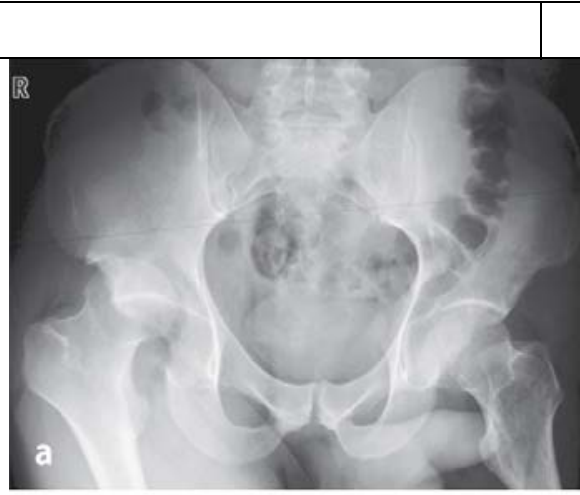

\section{Azetabulum/Becken}
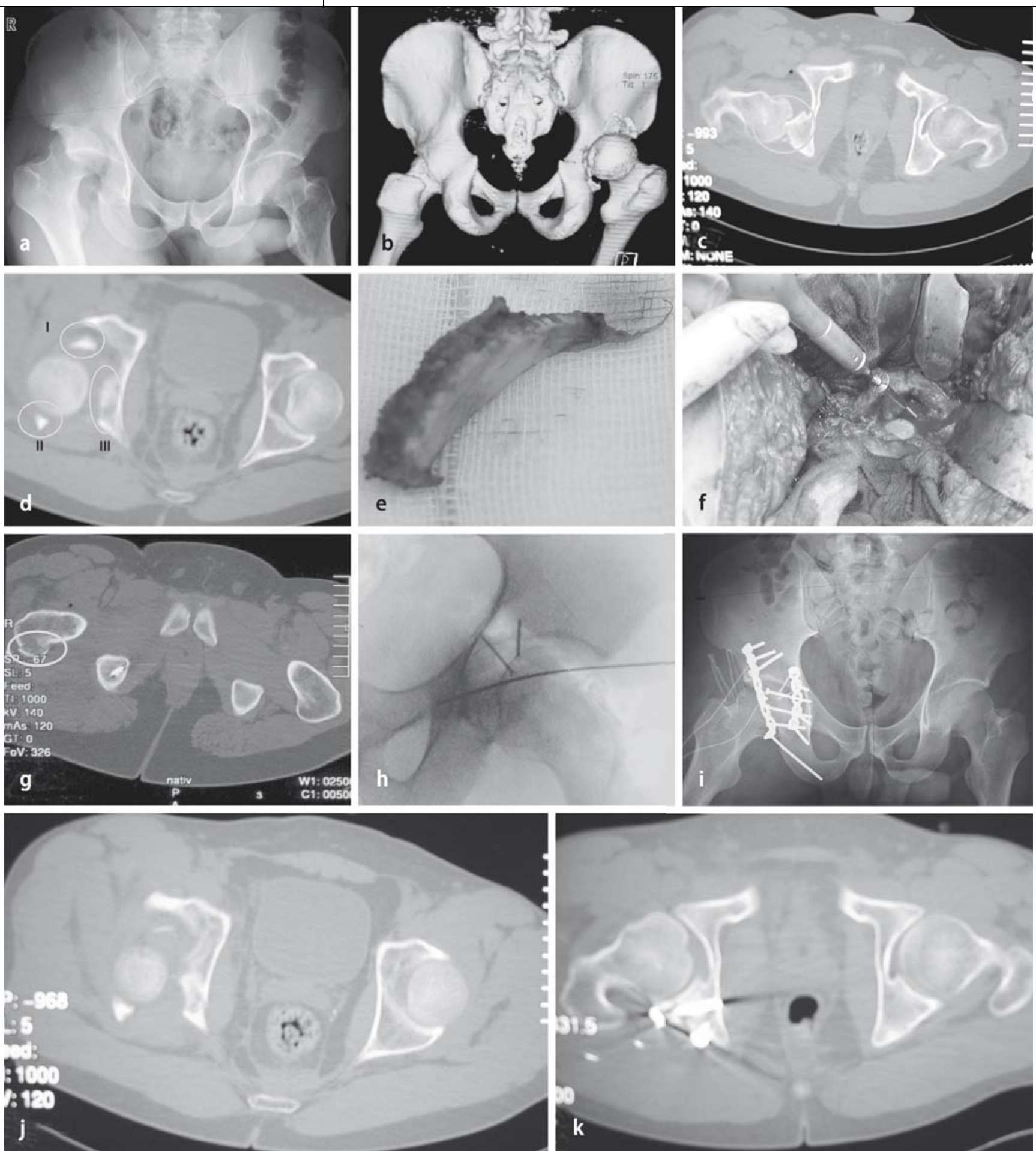

Abb. 6 \36-jähriger Patient, a a.-p. Aufnahme, Hüftluxationsfraktur, hinterer Pfeiler mit Fraktur des hinteren Walls, b 3D-Rekonstruktion, Zerstörung des hinteren Walls und Fraktur, wenig disloziert, des hinteren Pfeilers, $\mathbf{c}, \mathbf{d}$ CT, axialer Schnitt, $\mathbf{c}$ Impression des Femurkopfs, Femurkopf im dorsalen Pfannenanteil (Kreis) verhakt, d/ freies, intraartikuläres Fragment, // Fraktur des hinteren Walls, III marginale Impaktion der Gelenkanteile des hinteren Pfeilers, Gelenkkongruenz aufgehoben, e freies intraartikuläres Fragment, $\mathbf{f}$ intraoperative Fotografie:Verschraubung des freien Fragments mit 2,0-mm-Schraube, Auffüllung des Defekts dorsal der Schraube mit autologer Spongiosa vom dorsalen Trochanter major, g postoperatives Kontroll-CT, axialer Schnitt, Darstellung des Trochanter-major-Bereichs mit Knochendeckel (Ort der intraoperativen Spongiosaentnahme), h intraoperative Durchleuchtungsaufnahme, Gelenk anatomisch rekonstruiert, eingepasstes, freies Fragment durch 2 2,0$\mathrm{mm}$-Schrauben gehalten, hinterer Wall anatomisch reponiert und temporär für Durchleuchtungskontrolle vor definitiver Plattenosteosynthese mit 1,6-mm-Kirschner-Draht fixiert, i postoperative a.-p. Aufnahme des Beckens, anatomische Gelenkrekonstruktion, $\mathbf{j}, \mathbf{k} C \mathrm{CT}$, axiale Schnitte, $\mathbf{j}$ prä-, $\mathbf{k}$ postoperativ, stufenlose Wiederherstellung der Gelenkkongruenz 

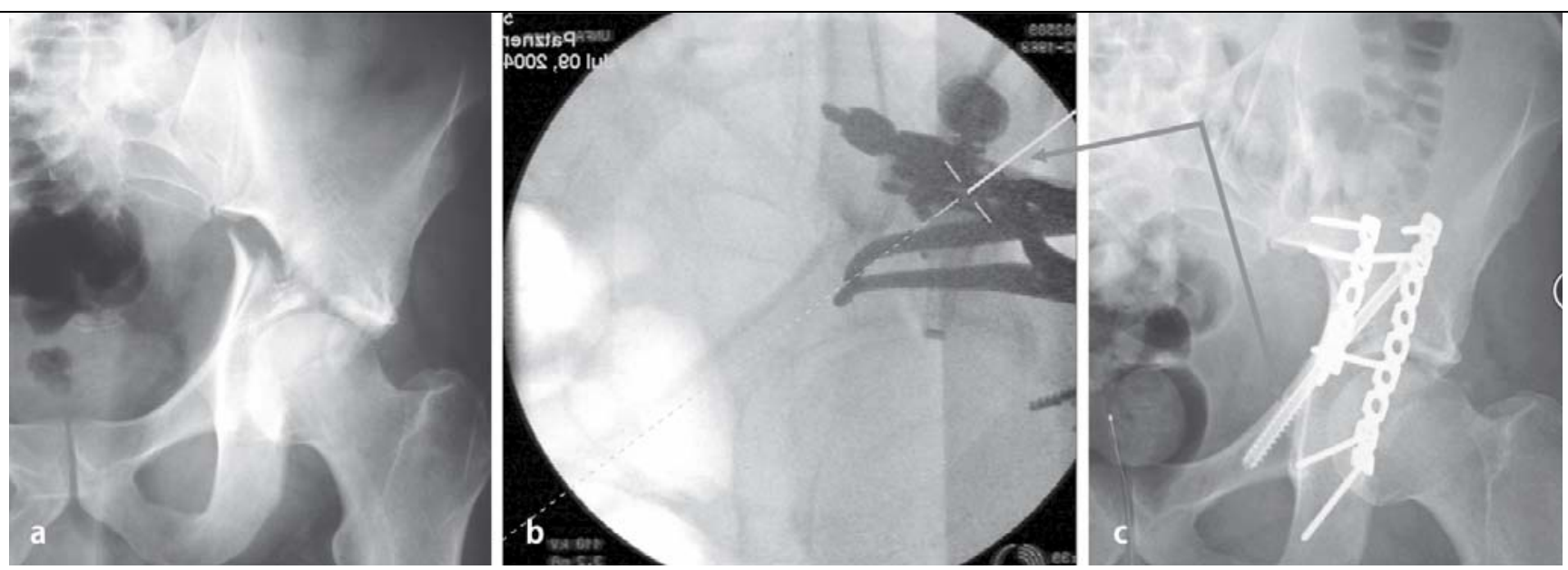

Abb. 7 \ 40-jähriger Patient, transtektale Querfraktur mit Beteiligung des hinteren Walls, a präoperative a.-p. Aufnahme, b intraoperative Durchleuchtung, Ausrichtung des navigierten Bohrers für die Zugschraube vom hinteren in den vorderen Pfeiler (Pfeil), c postoperative a.-p. Aufnahme, Stabilisierung des hinteren Pfeilers und des hinteren Walls mit 2 3,5-mm-Beckenrekonstruktionsplatten und durchbohrter Pfeilerschraube (Pfeil)

modellierte Platten zu versuchen, noch nicht 100\%ige Repositionsergebnisse beim Anziehen der ersten Schrauben über die aufgebrachte Platte definitiv zu reponieren. Ein wesentlich größeres Risiko liegt darin, dass mit dem Besetzen der Plattenlöcher die Fragmente über die nicht korrekt angepasste Platte wieder disloziert werden.

Allen 3 Zugängen ist zueigen,

- dass es „unsave zones“ gibt, in denen, wenn sie von Platten überbrückt werden, möglichst keine Schrauben in die Platten eingebracht werden sollten, um deren Penetration in das Gelenk zu vermeiden (insbesondere wichtig beim Ilioinguinalen Zugang, da hier klassischerweise das Gelenk zu keinem Zeitpunkt während der Operation eingesehen wird und die Schraubenlage nicht kontrolliert werden kann), und

- dass es Areale gibt, die sich für die Plattenlage besonders gut eignen, da in ihnen ein guter „bone stock“ vorhanden ist, in dem Schrauben gut greifen und auch möglichst lange Schrauben eingebracht werden können.

Beim Ilioinguinalen Zugang liegt folgende "unsave zone“ vor: vom Tuberculum innominatum ventral bis dorsal des Azetabulums, in etwa der dorsalen Begrenzung des entspannten $M$. iliopsoas entsprechend. Die Plattenlage sollte entlang der Linea terminalis des kleinen $\mathrm{Be}$ - ckens vom Tuberculum pubicum ventral bis zur Sakroiliakalfuge dorsal gewählt werden.

Beim Kocher-Langenbeck-Zugang bestehen „unsave zones“

- an der rechten Seite etwa zwischen 7 und $11 \mathrm{Uhr}$ an der dorsalen Gelenklippe und

- an der linken Seite von 1-5 Uhr an der dorsalen Gelenklippe.

Platten müssen unbedingt so anatomisch vorgebogen werden, dass sie möglichst nahe am Rand der Gelenklippe liegen, ohne ein Impingement auf das Labrum acetabuli auszulösen. Gleichzeitig sollten kranial und kaudal der "unsave zone" gebohrte Schrauben entweder nach kranial, Richtung Incisura ischiadica major, oder nach kaudal, Richtung ,ischial gutter", gebohrt werden. Schrauben, die innerhalb der „unsave zone" eingebracht werden, müssen sehr tangential gebohrt, auf ihren Halt in den Fragmenten überprüft und dürfen keinesfalls zu lang gewählt werden. Niemals sollte man hintere Wallfrakturen, selbst bei guter Knochenqualität und großen einzelnen Fragmenten, nur über das Einbringen von 3,5mm-Zugschrauben für die Fragmentfixation versorgen, da die Gefahr einer sekundären Dislokation zu groß ist. Neben den Zugschrauben sollte immer zusätzlich eine gebogene Beckenrekonstruktionsplatte aufgebracht werden, um die Fragmente, im Sinn eines „buttressings“, abzustützen.
Mechanisch größte Stabilität lässt sich über das Einbringen von Pfeilerschrauben zwischen dem vorderen und hinteren Pfeiler - bzw. umgekehrt - herbeiführen, wobei die Landmarken für den Eintritt, die Bohrrichtung und die intraoperative Durchführung des Bohrens ausführlich u. a. von Isler u. Ganz [13] beschrieben wurden. Dennoch ist das Platzieren dieser wichtigen Schrauben keineswegs ,einfach“. Deutlich erleichtert wurde die Operationstechnik dieser - teilweise recht langen - Pfeilerschraube durch die Möglichkeit, sie navigiert zu platzieren (• Abb. 7).

Am Ende der Operation muss immer eine abschließende, sorgfältige Röntgenkontrolle erfolgen. Dazu gehören die Becken-a.-p.- und die beiden Judet-Aufnahmen. Im Zweifelsfall, wenn es fraglich zu einer intraartikulären Schraubenlage gekommen ist, helfen auch Projektionen, die jeweils $45^{\circ}$ über- oder unterschreiten. Beantworten die über-/unterdrehten Aufnahmen die Frage nach der Gelenkpenetration durch eine/mehrere Schrauben nicht, muss man die Schraube kürzen, ganz entfernen oder neu bohren. Es wird dringend empfohlen, die intraoperativen Durchleuchtungsaufnahmen sofort auszudrucken und der Operationsdokumentation beizulegen. Stellen sich bei der abschließenden intraoperativen Röntgenkontrolle ein unbefriedigendes Repositions- und Fixationsergebnis dar, hat der Operateur im Grund keine Alternativen, als folgenden Katalog abzufragen: 
- Kann er selbst akut ein besseres Ergebnis, nach Auflösen der Osteosynthese herbeiführen?

- Braucht er Hilfe?

- Ist die Knochenqualität noch ausreichend, um erneut die Fragmente zu lösen und zu manipulieren?

- Und - am allerwichtigsten - hat er eine Vorstellung, warum die Reposition/Fixation unzureichend ist?

Prinzipiell ist es schwer, Empfehlungen für diese Situation abzugeben, da sie nicht auftreten sollte.

\section{Wundverschluss}

Nach der Durchleuchtungskontrolle erfolgt der anatomische Wundverschluss. Wir entnehmen routinemäßig nach Beckenosteosynthesen, vor dem ausgiebigen Spülen mit minimal 11 Ringer-Lösung, mindestens 2 Abstriche aus der Tiefe. Nach dem Spülen dränieren wir beim ilioinguinalen Zugang jedes Fenster einzeln, beim Kocher-Langenbeck-Zugang legen wir nach der Rekonstruktion der kleinen Außenrotatoren eine Dränage submuskulär unter dem M. glutaeus maximus ein. Eine weitere Dränage liegt jeweils subfaszial, und insbesondere beim Kocher-Langenbeck-Zugang sollte, bei kräftigem subkutanem Gewebe, eine subkutane Dränage platziert werden. Bei allen Patienten wird am Ende der Operation, nach dem Wundverschluss, ein Beckenspica angelegt, der für 5-7 Tage auch nach den Verbandswechseln auf den Stationen jeweils erneut angewickelt wird.

\section{Nachbehandlung/ Komplikationsprophylaxe}

Die Nachbehandlung setzt sich zusammen aus:

- Fortführung der Antibiotikaprophylaxe für 3, maximal 5 Tage (erweiterte Zugänge)

- Beginn der Prophylaxe heterotoper Ossifikationen am Operationstag mit 2-mal $75 \mathrm{mg}$ Indomethacin p.o. oder rektal für 14, maximal 28 Tage bei den erweiterten und dem Kocher-Langenbeck-Zugang

- CT Kontrolle des Beckens am 1. postoperativen Tag $[5,20,21]$
- Bettruhe für 2 Tage

- Entfernen der Dränagen am 2., spätestens am 3. postoperativen Tag, im Verlauf Verbandswechsel alle 2 Tage, bis die Hautfäden nach 12-16 Tagen gezogen sind

- Beginn der physikalischen Therapie mit Krankengymnastik des Hüftgelenks aktiv-assistiert und Mobilisation mit $5 \mathrm{~kg}$ Teilbelastung nach der Entfernung der Redon-Dränagen; 5 bis maximal $10 \mathrm{~kg}$ Teilbelastung werden konsequent für insgesamt 6 Wochen aufrecht erhalten, erst danach beginnt der zunehmende Belastungsaufbau bis zur Vollbelastung (siehe z. B. Russell et al. [23]).

- Konventionelle Röntgenkontrollen (a.-p. und Judet-Aufnahmen) am Tag der Verlegung in die Rehabilitationsklinik und im Verlauf, während der ersten 3 Monate im Abstand von etwa 4 Wochen

Insbesondere bei den erweiterten Zugängen und vereinzelt beim Ilioinguinalen Zugang kann es zum Austritt von seröser Flüssigkeit aus der Operationswunde oder den Redon-Dränagen-Austrittsstellen aus der Haut kommen. Es ist unsere erklärte Politik, dass wir, wenn die Sekretion aus der Wunde nach 5 Tagen nicht vollständig versiegt ist, die Antibiotikaprophylaxe fortführen und nach 7 Tagen, wenn die Wunde zu diesem Zeitpunkt nicht absolut trocken ist, einen „wash out“ durchführen, selbst bei ansonsten äußerlich unauffälliger Wunde, negativen postoperativen Abstrichen der Erstoperation und normalen Entzündungsparametern (CRP zu diesem frühen Zeitpunkt postoperativ meist noch auf etwa 70 U/l erhöht, Leukozyten <10.0oo).

„Wash out" bedeutet, dass sämtliche Fäden in der Tiefe entfernt, alle Fenster eröffnet und das Plattenlager dargestellt werden. Nach Serienabstrichen erfolgt eine JetLavage mit 51 Ringer-Lösung, gefolgt von erneuten Serienabstrichen. Dränagen werden wie in der Erstoperation eingelegt, und es erfolgt der anatomischem Wundverschluss. Auf diese Weise konnten wir unsere Infektionsrate in den letzten 8 Jahren bei weit mehr als 200 operierten Beckenund Azetabulumfrakturen - bei Patienten mit primär intakten Weichteilen - auf insgesamt 3 Patienten beschränkt halten.

\section{Resümee}

Dislozierte Azetabulumfrakturen müssen anatomisch, ohne Stufen- und Spaltbildung, rekonstruiert werden, um ein schmerzfreies, belastbares, langzeitüberlebendes Gelenk wieder herzustellen. Aufgrund der typischen Frakturmuster, der Lage des Azetabulums in der Tiefe des Beckens und der hohen mechanischen Belastung, der das Hüftgelenk ausgesetzt ist, sind ein tiefes Verständnis der Anatomie des Azetabulums/Beckens, der Frakturklassifikation nach Judet/Letournel, der anatomischen Zugangswege mit ihren Möglichkeiten und den sehr großen Risiken v. a. für den weniger erfahrenen Operateur absolute Grundvoraussetzung, um anatomisch reponieren und fixieren zu können. Azetabulumfrakturen sollten daher in Zentren operiert werden, da nur dort in Bezug auf Logistik, Erfahrung und Möglichkeit auch für die Komplikationsbeherrschung ausreichende Voraussetzungen gegeben sind. Diese Forderung ergibt sich letztendlich bereits aus der Tatsache, dass die Zahl der pro Jahr in Deutschland zu versorgenden Becken- und Azetabulumfrakturen bei nur etwa 4000 Fällen liegt. Es macht daher weder für den einzelnen betroffenen Patienten noch für den einzelnen betroffenen Operateur Sinn, im Jahr 3-5 dieser Frakturen zu versorgen, da unter diesen Voraussetzungen die Ergebnisse nicht einmal hinreichend sein werden. Die vorhandenen Statistiken zeigen eindrücklich, dass nur nach langer persönlicher Lernkurve und großen Zahlen versorgter Patienten mit hoher Wahrscheinlichkeit exzellente Ergebnisse zu erwarten sind.

\section{Korrespondierender Autor Prof. Dr. J. M. Rueger}

Klinik für Unfall-, Hand- und Wiederherstellungschirurgie, Zentrum für Operative Medizin, Universitätsklinikum Hamburg-Eppendorf Martinistraße 52, 20246 Hamburg jmrueger@uke.uni-hamburg.de

Interessenkonflikt. Es besteht kein Interessenkonflikt. Der korrespondierende Autor versichert, dass keine Verbindungen mit einer Firma, deren Produkt in dem Artikel genannt ist, oder einer Firma, die ein Konkurrenzprodukt vertreibt, bestehen. Die Präsentation des Themas ist unabhängig und die Darstellung der Inhalte produktneutral. 


\section{Literatur}

1. Adam P, Labbe JL, Alberge Y et al. (1985) The role of computed tomography in the assessment and treatment of acetabular fractures. Clin Radiol 236: 13-18

2. Beaule PE, Dorey FJ, Matta JM (2003) Letournel classification for acetabular fractures. Assessment of interobserver and intraobserver reliability. J Bone Joint Surg Am 85A: 1704-1709

3. Berner M, Ulrich C (1999) Die primäre endoprothetische Versorgung von Acetabulumfrakturen. Osteo Int 7: 93-100

4. Borelli J, Goldfarb C, Catalano L et al. (2002) Assessment of articular fragment displacement in acetabular fractures: a comparison of computerized tomography and plain radiographs. J Orthop Trauma 16: 449-456

5. Borrelli J, Ricci WM, Steger-May K et al. (2005) Postoperative radiographic assessment of acetabular fractures. J Orthop Trauma 19: 299-304

6. Brandser E, Marsh JL (1998) Acetabular fractures: easier classification with a systematic approach. Am J Roentgenol 171: 1217-1228

7. Burkhardt M, Gänsslen A, Uder M et al. (2003) Neue Möglichkeiten der Visualisierung von Frakturen mittels CT: Rekonstruktionen, 3D-Planungen. Zentralbl Chir 128: 34-39

8. Fishman AJ, Greeno RA, Brooks RL et al. (1994) Prevention of deep vein thrombosis and pulmonary embolism in acetabular and pelvic fracture surgery. Clin Orthop 305: 133-137

9. Hak DJ, Hamel AJ, Bay BK et al. (1989) Consequences of transverse acetabular fracture malreduction on load transmission across the hip joint. J Orthop Trauma 12: 90-100

10. Harley JD, Mack LA, Winquist RA (1982) CT of acetabular fractures: comparison with conventional radiography. AJR Am J Roentgenol 138: 413-417

11. Helfet DJ, Schmeling GJ (1994) Management of complex acetabular fractures through single nonextensile exposures. Clin Orthop 305: 58-68

12. Hüfner T, Pohlemann T, Gänsslen A et al. (1999) Die Wertigkeit der CT zur Klassifikation und Entscheidungsfindung nach Acetabulumfrakturen. Unfallchirurg 102: 124-131

13. Isler B, Ganz R (1990) Classification of pelvic girdle injuries. Unfallchirurg 93: 289-302

14. Kellam J, Messer A (1994) Evaluation of the role of coronal and sagittal axial CT scan reconstructions for the imaging of acetabular fractures. Clin Orthop 305: 152-159

15. Letournel $E$, Judet $R$ (1993) Radiology of the normal acetabulum. In: Letournel $E$, Judet R (eds) Fractures of the acetabulum, 2nd edn. Springer, Berlin Heidelberg New York

16. Matta JM (1994) Operative treatment of the acetabular fractures through the ilioinguinal approach a ten years perspective. Clin Orthop 305: 10-19

17. Matta JM (1996) Fractures of the acetabulum: accuracy of reduction and clinical results in patients managed operatively within three weeks after the injury. J Bone Joint Surg Am 78A: 1632-1645

18. Matta JM, Anderson LM, Epstein AC et al. (1986) Fractures of the acetabulum: a retrospective analysis. Clin Orthop 215: 230-240

19. Mears D, Velyvis J, Chang CP (2003) Displaced acetabular fractures managed operatively: indicators of outcome. Clin Orthop 407: 173-186

20. Moed BR, Carr SE, Watson JT (2000) Open reduction and internal fixation of posterior wall fractures of the acetabulum. Clin Orthop 377: 57-67
21. Moed BR, Carr SEW, Gruson KI et al. (2003) Computed tomographic assessment of fractures of the posterior wall of the acetabulum after operative treatment. J Bone Joint Surg Am 85: 512-522

22. Olson SA, Matta JM (1993) The computerized tomography subchondral arc: a new method of assessing acetabular articular continuity after fracture (a preliminary report). J Orthop Trauma 7: 402-413

23. Russell G, Nork S, Chip Routt MA (2001) Perioperative complications associated with operative treatment of acetabular fractures. JTrauma 51: 10981103

24. Stroszcynski C, Schedel H, Stöckle U et al. (1996) Klinischer Einsatz multiplanarer und 3D-Rekonstruktionen der Spiral-CT in der Diagnostik der Acetabulumfrakturen. Aktuelle Radiol 6: 91-95

25. Wright R, Barrett K, Christie MJ et al. (1994) Acetabular fractures: long term follow-up of open reduction and internal fixation. J Orthop Trauma 8: 397-403 